\title{
EchoGéo
}

$21 \mid 2012$

Pays émergents

\section{Pratiques d'une métropole émergente par les usagers des transports en commun, le cas du Cap, Afrique du Sud}

\section{Solène Baffi}

\section{CpenEdition}

Journals

Édition électronique

URL : https://journals.openedition.org/echogeo/13139

DOI : 10.4000/echogeo.13139

ISSN : 1963-1197

Éditeur

Pôle de recherche pour l'organisation et la diffusion de l'information géographique (CNRS UMR 8586)

Référence électronique

Solène Baffi, «Pratiques d'une métropole émergente par les usagers des transports en commun, le cas du Cap, Afrique du Sud », EchoGéo [En ligne], 21 | 2012, mis en ligne le 10 octobre 2012, consulté le 10 août 2021. URL : http://journals.openedition.org/echogeo/13139 ; DOI : https://doi.org/10.4000/ echogeo.13139

Ce document a été généré automatiquement le 10 août 2021.

EchoGéo est mis à disposition selon les termes de la licence Creative Commons Attribution - Pas d'Utilisation Commerciale - Pas de Modification 4.0 International (CC BY-NC-ND) 


\title{
Pratiques d'une métropole émergente par les usagers des transports en commun, le cas du Cap, Afrique du Sud
}

\author{
Solène Baffi
}

\section{Introduction}

1 En Juin 2007, la municipalité du Cap approuvait dans son plan de transport quinquennal la mise en place du Bus Rapid Transit, équivalent du Bus à Haut Niveau de Service. Ce nouveau mode de transport, également installé à Johannesburg, est censé répondre aux mutations qui affectent la métropole émergente du Cap. Ces mutations, tant démographiques, économiques, que sociales, sont d'autant plus exacerbées dans un pays où les effets de la transition post-apartheid demeurent particulièrement visibles. La volonté d'installer ce nouveau mode de transport répond alors à un double objectif. Il s'agit d'une part d'accompagner ces mutations, aussi bien en contrôlant la diffusion de l'urbanisation dans une ville en proie à l'étalement urbain qu'en assurant l'accessibilité et les mobilités des citadins. Il s'agit d'autre part de pourvoir la métropole d'un mode de transport public moderne, afin de pouvoir rivaliser avec les métropoles internationales, le Cap prétendant devenir une «ville globale (Baffi, 2010). Toutefois, si le service du BRT a été inauguré en 2010, les principales lignes du bus, notamment celles assurant la liaison entre le principal township de Khayelitsha et le centre-ville, ne sont toujours pas achevées.

2 La nécessité d'un nouveau mode de transport public en Afrique du Sud est par ailleurs à mettre en lien avec la persistance d'une ségrégation liée aux décennies de politique d'apartheid. Alors que la politique d'apartheid a pu être décrite comme une «mise en scène de la distance » (Houssay-Holzschuch, 1996), les populations stigmatisées par les politiques ségrégatives étaient physiquement exclues de la ville. Or ces populations 
habitent encore aujourd'hui majoritairement dans les townships excentrés des bassins d'emplois et du centre-ville, principal bassin d'emplois (illustration 1). Les usagers des transports collectifs demeurent essentiellement les populations les plus pauvres, contraintes d'utiliser des services de transport - de bus, minibus, ou train - coûteux, longs et faiblement intégrés entre eux. Toutefois, en 2004, les déplacements effectués dans les transports publics ne représentaient que $39 \%$ des déplacements, contre $48 \%$ effectués dans les véhicules privés (ITP 2006). L'importance du parc de véhicules privés est significatif de la dualité qui caractérise encore la société sud-africaine ; si la majorité des classes moyennes et supérieures possèdent une voiture, les classes inférieures, résidant le plus souvent encore dans les townships, demeurent dépendantes des transports publics. L'importance du trafic des véhicules privés implique des nuisances telles que des problèmes de congestion ou de pollution, et contribue également à renforcer l'étalement urbain.

Illustration 1 - Localisation des investissements privés et des projets d'habitat social au Cap : les townships à l'écart des bassins d'emplois et des zones d'activités

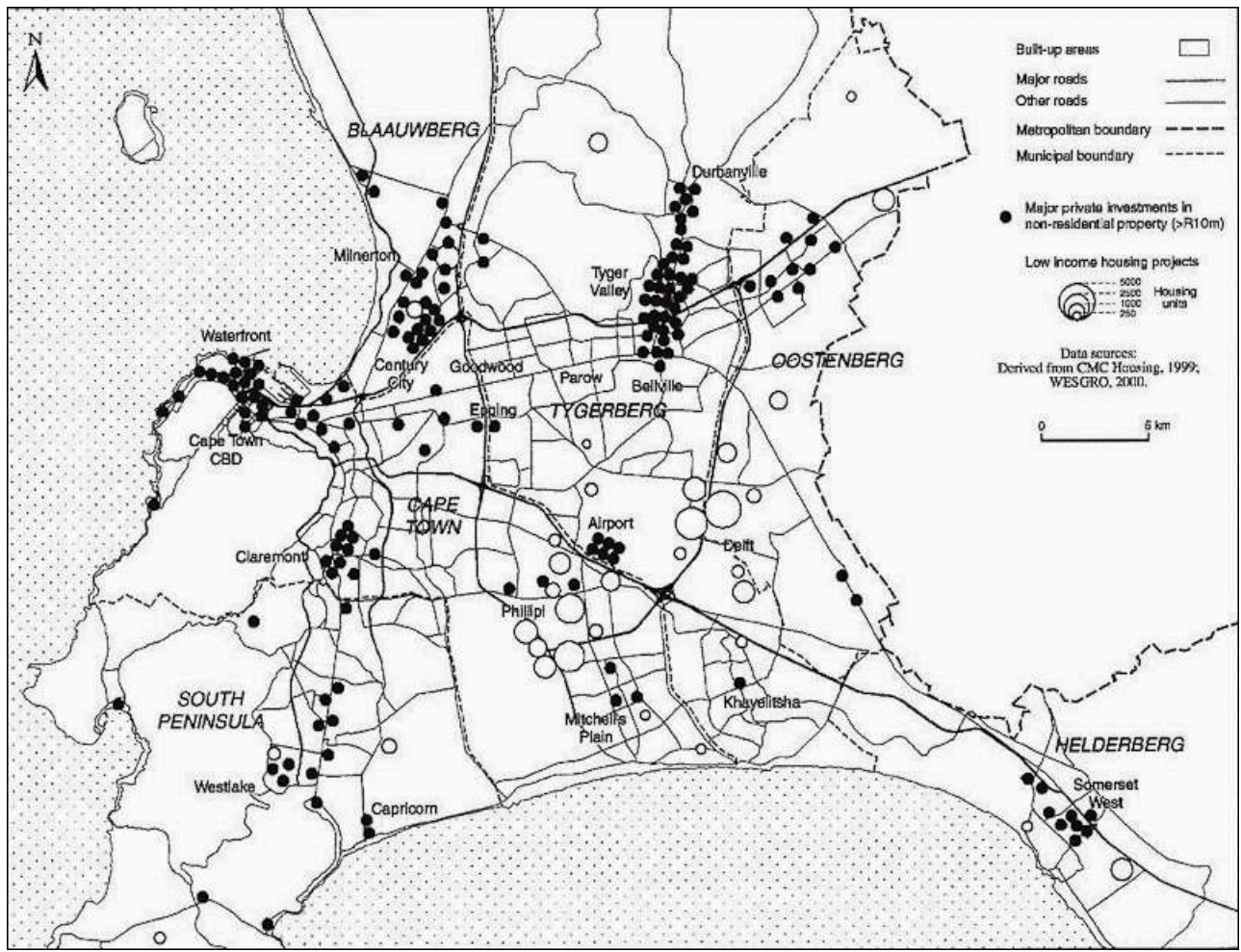

Source : Turok, Watson, 2001

Pour les usagers des transports en commun, les mobilités sont d'autant plus contraignantes qu'elles sont renforcées par le spatial mismatch hérité de l'apartheid (Naudé, 2008). Il en résulte une distorsion entre zones d'emploi et zones de résidence de la main d'œuvre et donc des distances importantes à franchir quotidiennement pour les près de $30 \%$ de citadins du Cap qui vivent dans les townships et les camps de squatters (Bénit, 1998). Ainsi, le dysfonctionnement urbain planifié par le régime d'apartheid a isolé durablement une grande partie de la population des zones d'activités urbaines, la contraignant à des déplacements quotidiens longs et coûteux. Les déplacements domicile-travail sont en effet nettement plus longs en Afrique du Sud que dans le reste du monde, notamment parmi les voyageurs des classes inférieures 
(Behrens, Wilkinson in Huchzermeyer, 2003). La distance moyenne des trajets domiciletravail s'établissait en 1999 autour de $20 \mathrm{~km}$ en Afrique du Sud, contre $11 \mathrm{~km}$ par exemple en Europe. Pour les usagers des transports publics, la question des mobilités et des conditions de transport est au cœur de leur quotidien.

Néanmoins ces mobilités évoluent, en lien avec le processus d'émergence et la transition post-apartheid qui animent la ville. Cette transition s'est accompagnée d'un nouveau dynamisme économique, social et politique, avec des implications tant sur l'emploi et la structure économique, avec notamment une transition vers le tertiaire, sur le nouveau jeu démocratique, que sur les pratiques sociales, en particulier liées aux modes de consommation. De ce fait, la ville sud-africaine s'éloigne peu à peu du modèle de la ville d'apartheid pour se rapprocher de celui de la ville émergente où les shoppings malls jouxtent parfois les zones d'habitat auto-construit. Parmi les citadins qui naviguent quotidiennement entre les différents territoires de la ville du Cap, les maids ${ }^{1}$ cristallisent ces différentes évolutions. D'une part, la catégorie socio-professionnelle des domestic workers à laquelle elles appartiennent, correspond généralement aux classes moyennes inférieures et tend à augmenter, puisqu'entre Janvier 2011 et Janvier 2012 leur nombre est passé de 877000 à 896000 en Afrique du Sud (Stattsa, 2012), à l'instar d'autres pays émergents (Bret, 2011). D'autre part, ces femmes effectuent quotidiennement des mobilités depuis les townships vers les banlieues huppées de la ville où elles sont employées. Elles sont dans cette mesure dépendantes des transports publics et effectuent des mobilités contraignantes. Toutefois, elles renouvellent également leurs mobilités en intégrant de nouvelles pratiques telles que les arrêts au shopping mall par exemple. Ainsi, la pratique des transports publics par cette catégorie de femmes apparait représentative des mutations qui affectent la ville du Cap, tant relatives à la transition post-apartheid qu'au processus d'émergence.

Dans quelle mesure l'évolution du système de transports publics et des mobilités traduit-elle cette tension urbaine d'une métropole en proie à de fortes disparités sociospatiales ? Comment les usagers, en particulier les maids, se positionnent-ils face à un service en grande partie hérité de la période d'apartheid et à bien des égards, dépassé ? Pour répondre à ces questions, cet article s'attache à mettre en évidence les mutations socio-spatiales de la ville au prisme des transports publics et de leurs pratiques par les usagers. Pour cela la première partie étudie la façon dont les transports publics capetowniens perpétuent tout autant qu'ils pallient cette situation de spatial mismatch. À partir de cette observation du système de transport, les mobilités d'un groupe précis d'usagères, les maids ${ }^{2}$, est plus particulièrement détaillée pour souligner la tension entre pratiques contraintes des transports publics, et stratégies d'adaptation. Enfin, l'échelle de l'analyse se resserre pour se concentrer sur le lieu du transport public, celui du bus par exemple dans le cas des maids, où se reflètent certaines caractéristiques de la société capetownienne dans son ensemble.

\section{Un système de transport hérité ou adaptatif ?}

6 Parmi les trois millions de citadins du Cap, la majorité utilisent le transport privé au quotidien, traduisant l'importance de la «car culture» (Urry, 1999). Toutefois, la possession de véhicules privés n'est pas homogène au sein de la société sud-africaine. Le ratio de 178 véhicules pour 1000 habitants est certes bien supérieur à celui observé dans la plupart des pays émergents et en développement, mais il ne représente qu'un 
tiers du ratio observé dans les pays européens, qui était par exemple de 570 véhicules pour 1000 habitants en Allemagne en 1998 (Behrens, Wilkinson, 2003). Et ce ratio s'applique de façon très disparate à l'échelle de l'agglomération capetownienne ; si $93 \%$ des habitants de Durbanville, une banlieue huppée du Cap, utilisaient un mode de transport privé en 2003, $85 \%$ des habitants de Khayelitsha utilisaient les transports publics (ITP, 2006). Le choix d'un transport privé pour effectuer des mobilités quotidiennes demeure donc un privilège réservé aux plus aisés, bien que les nouvelles classes moyennes tendent à grossir les rangs de ces usagers.

$7 \quad$ Les usagers dépendant des transports collectifs font ensuite le choix entre trois modes de transport: le train, le bus et le minibus. Ces trois modes de transport se sont développés indépendamment et à des époques différentes, offrant aujourd'hui un réseau et des infrastructures peu voire pas intégrés et un service segmenté. Le réseau ferré intra-urbain a été mis en place dès la fin du XIX ${ }^{e}$ siècle au Cap vers les banlieues est et sud, entraînant le développement résidentiel de ces banlieues. Le réseau radial aujourd'hui proposé prend en charge la majorité de la population usagère des transports publics, notamment sur la ligne reliant Khayelitsha au centre-ville. Cette fréquentation peut s'expliquer par le faible coût de ce mode de transport, également perçu comme le plus dangereux en heure de pointe en raison du nombre de pickpockets et d'agressions qui se déroulent dans les transports. C'est pourquoi un certain nombre d'usagers, en particulier les femmes, préfèrent utiliser le bus. La principale compagnie de Golden Arrow et la petite compagnie de Sibanye assurent quotidiennement $18 \%$ du transport de passagers, principalement entre les townships et les zones d'emploi. Le réseau a été mis en place pendant l'apartheid afin d'assurer les mobilités de travail des habitants des townships. À ce jour encore, ce mode de transport prend essentiellement en charge des travailleurs, aussi bien sur des longues que des courtes distances mais avec un coût plus élevé que le train.

\begin{tabular}{|l|l|}
\hline \multicolumn{2}{|l|}{ Prix d'un trajet sur la ligne Le Cap-Khayelitsha (en Rands) } \\
\hline train & 3 (avec une clipcard mensuelle) \\
\hline bus & $\begin{array}{l}7,10 \text { (avec une clipcard mensuelle) } \\
7,75 \text { (avec une clipcard hebdomadaire) }\end{array}$ \\
\hline minibus & 12 \\
\hline
\end{tabular}

Les minibus transportent $29 \%$ des usagers quotidiens des transports en commun. Ce mode de transport s'est développé sur le modèle de la petite entreprise au sein des townships pendant l'apartheid, au point de devenir indispensable, ce qui a amené le gouvernement à entreprendre une politique de régulation du secteur à partir de 1994. Malgré le succès mitigé de cette politique ${ }^{3}$, les minibus restent un mode de transport extrêmement populaire disposant du réseau le plus développé à l'échelle de la métropole.

Ces trois modes de transports collectifs acheminent la population depuis le centre-ville jusque dans les différentes banlieues et entre ces banlieues qui sont souvent lointaines (l'agglomération du Cap s'étend sur près de $2500 \mathrm{~km}^{2}$ ). Mais la couverture de la métropole est très inégale : certaines zones demeurent peu voire pas desservies par les 
réseaux de transports, comme le nord-est de l'agglomération autour de Brackenfell, la côte ouest à hauteur de Big Bay ou encore à l'est Somerset West (illustration 2). S'il y a certes une complémentarité entre le réseau de minibus et les autres réseaux, les minibus jouant un rôle de feeder vers les gares ferroviaires et les arrêts de bus, celle-ci demeure partielle puisqu'elle n'est assurée que par les minibus, et uniquement dans la mesure où ceux-ci s'adaptent à la demande. Et lorsque la convergence des différents réseaux est assurée, l'absence d'interconnexion et de multimodalité ne permet pas à l'usager d'en tirer parti : il doit, pour changer de transport, changer d'infrastructures (or celles-ci peuvent parfois être à plusieurs centaines de mètres les unes des autres), et s'acquitter d'un autre titre de transport.

Illustration 2 - Localisation des différents suburbs de la métropole du Cap

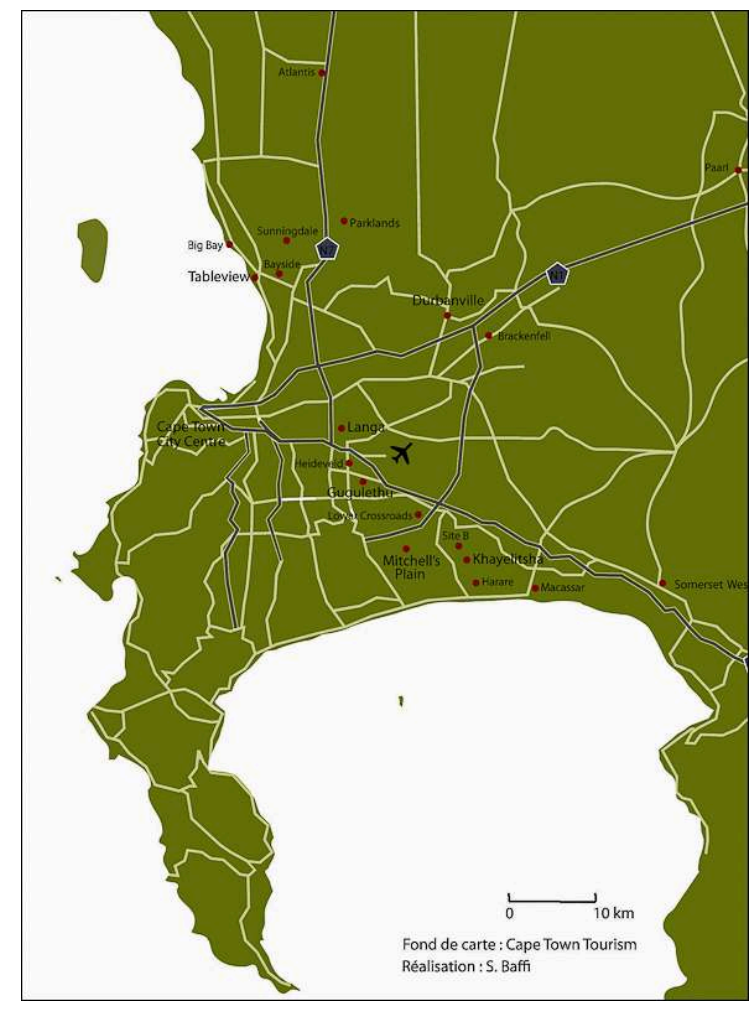

Source : Cape Town Tourism (fond de carte) ; réalisation : S. Baffi.

Face à de telles difficultés pour effectuer des mobilités quotidiennes, on comprend que pour les populations dépendant des transports publics le déplacement est avant tout une nécessité. La majorité des usagers effectuent des mobilités de travail qui sont d'autant plus incompressibles que le taux de chômage atteint plus de $50 \%$ de la population des townships du Cap d'âge actif (Nitrass, 2002). Toutefois, on peut noter plusieurs évolutions dans la desserte des différents réseaux de transport depuis le début des années 1990. Ces évolutions sont à mettre en lien avec des mutations sociales plus larges, notamment la croissance des mobilités de consommation au sein des townships, où fleurissent les shopping malls depuis plusieurs années. Les clients de ces lieux de consommation appartiennent le plus souvent aux catégories des lower income ${ }^{4}$ de l'agglomération, ils se déplacent en transport en commun et accèdent à la consommation souvent grâce à la politique des grants ${ }^{5}$ qui a permis d'améliorer le niveau de vie des habitants. Or les différents services de transports publics tendent à 
répondre à ces évolutions, même si cela se solde parfois par des actes de violence. Ce fut le cas par exemple en 2009, lorsqu'un nouveau centre commercial a ouvert ses portes dans une banlieue nord du Cap. Les deux associations de minibus présentes dans ce quartier se sont livré une guerre ouverte alors que la municipalité était incapable de mener à bien les négociations.

11 Les mutations démographiques à l'œuvre dans l'agglomération, liées en grande partie à l'afflux de migrants d'Afrique australe, contribuent également à influencer les dessertes de transports. Ces migrants contribuent à grossir les rangs des camps de squatters qui se développent dans les anciennes buffer zones de l'agglomération, en particulier en périphérie de Khayelitsha. Si le secteur de minibus, étant donné sa flexibilité, peut rapidement s'adapter à la présence de cette nouvelle clientèle, il en va autrement en ce qui concerne le réseau de bus. La compagnie Golden Arrow, qui détenait le monopole du service de bus pendant l'apartheid, est en effet toujours soumise aux contrats alors en vigueur. La compagnie est encore aujourd'hui subventionnée uniquement sur les trajets qui étaient reconnus comme indispensables aux mobilités des travailleurs des townships. La marge d'action de Golden Arrow se restreint désormais à l'ouverture de dessertes supplémentaires sur les trajets déjà assurés, sous réserve de l'accord de la municipalité. Les forums organisés tous les mois par la compagnie avec ses usagers sont donc l'occasion de recenser les nouveaux arrêts nécessaires pour accompagner l'urbanisation croissante des périphéries et interstices de l'agglomération. À l'intérieur même des townships, une même volonté d'étendre les réseaux est visible, en particulier avec l'extension de la ligne ferroviaire intra-urbaine à Khayelitsha, au sein d'un quartier en voie de rénovation et de formalisation (Ninot, Musil, Baffi, Drevelle, 2012).

Les populations des lower income du Cap sont ainsi les principales usagères des services de transport public, en particulier pour se rendre sur leur lieu de travail. Les systèmes de transport, par train, bus ou minibus, répondent à ces mobilités quotidiennes en offrant une desserte encore sclérosée, symptomatique des politiques urbaines héritées de l'apartheid. Mais les évolutions des différents réseaux laissent entrapercevoir les mutations tant sociales, démographiques et économiques qui construisent désormais la métropole et auxquels les différents services de transport doivent s'adapter (Baffi, 2010).

\section{Le parcours des maids, entre contrainte et appropriation}

13 Les pratiques des transports publics par leurs usagers ont évolué en lien avec les mutations politiques, économiques et sociales qu'a connu le pays, en particulier depuis 1994. Cela est tout à fait visible dans le cas des maids. La profession de ces femmes a évolué, tout d'abord en raison de la flexibilisation du travail. Si elles étaient auparavant le plus souvent au service d'une famille chez laquelle elles résidaient en raison des restrictions de déplacement des populations non-blanches, elles se rendent désormais quotidiennement au lieu de résidence des particuliers, qui peut d'ailleurs varier en fonction des jours de la semaine. Le nombre d'employeurs se multiplie en effet au fur et à mesure que la classe moyenne s'accroît. Il en résulte, au sein de cette profession, des mobilités diffuses et des pratiques sociales en cours de recomposition, à mettre en lien avec l'ouverture économique, politique et sociale du pays (Bénit, Morange, 2004). 

mutations économiques qu'a connu l'Afrique du Sud ces dernières décennies. Le passage au post-fordisme marque bien, en effet, la fin du fordisme « racial», mais il s'est également traduit par un rapide déclin des emplois industriels et une montée du chômage. L'essor du secteur informel et des services est l'une des conséquences de cette évolution, dont le travail domestique est un bon exemple (Bénit, Morange, 2004). Par ailleurs, en Afrique du Sud comme dans d'autres pays, l'accroissement du nombre d'employés domestiques correspond en partie à l'importance des migrations villecampagne (Anderfuhren, 2002). Le secteur informel est souvent le premier recours pour les nouveaux arrivants à la ville, comme c'est le cas des nombreuses femmes qui font régulièrement le choix de quitter la province rurale de l'Eastern Cape voisine pour venir tenter leur chance au Cap. multiplication récente des agences de recrutement et des associations de maids, révèle le passage de cette profession souvent informelle et peu reconnue à une forme moderne de travail domestique (Varrel, 2002). Cette formalisation est d'autant plus symbolique dans le cas du travail domestique qui est généralement associé aux catégories sociales défavorisées, que ce soit en Inde avec les Intouchables (Varrel, 2002), au Yémen avec les akhdâm (Destremeau, 2002), ou en Afrique du Sud avec la population noire jusqu'à la fin de l'apartheid. Si les salaires étaient souvent indigents, la reconnaissance progressive de cette profession et la mobilité sociale maintenant associée a permis aux maids d'atteindre bien souvent la catégorie des lower middle class ${ }^{6}$. Les salaires s'échelonnent en effet entre 300 et 2400 Rands au sein de la profession, avec un revenu médian de 1000 Rands (Stattsa, 2010), assurant à ces employés un niveau de vie modeste mais non pas indigent. La forte mobilité sociale et économique de ce corps de métier ces dernières années permet donc de souligner certaines caractéristiques propres au processus d'émergence.

Les maids constituent l'une des catégories d'usagers quotidiens des transports publics; elles réalisent chaque jour des déplacements entre les townships vers des banlieues surburbaines huppées, ou en direction du CBD du Cap. Il est tout d'abord clairement apparu que les mobilités pendulaires réalisées par les maids sont des mobilités longues et fastidieuses, coûteuses tout autant en temps qu'en argent. Le budget temps de ces femmes varie de une à deux heures par trajet juste pour se rendre sur leur lieu de travail en raison de la faible vitesse des bus, $20 \mathrm{~km} / \mathrm{heure}$ en moyenne. Beaucoup d'entre elles se lèvent entre 5 et 6 heures tous les jours car il faut, avant le transport, se rendre à l'arrêt de bus et prévenir les fréquents retards des bus dus aux retards des chauffeurs et aux embouteillages. On peut en effet remarquer que les horaires des bus, censés être fixes, sont souvent aléatoires (illustration 3).

EchoGéo, 21 | 2012 
Illustration 3 - Horaires de bus observés le $18 / 02 / 2010$ et le $2 / 03 / 2010$ entre $16 \mathrm{~h} 30$ et $17 \mathrm{~h} 15$ à l'arrêt de bus de Bayside

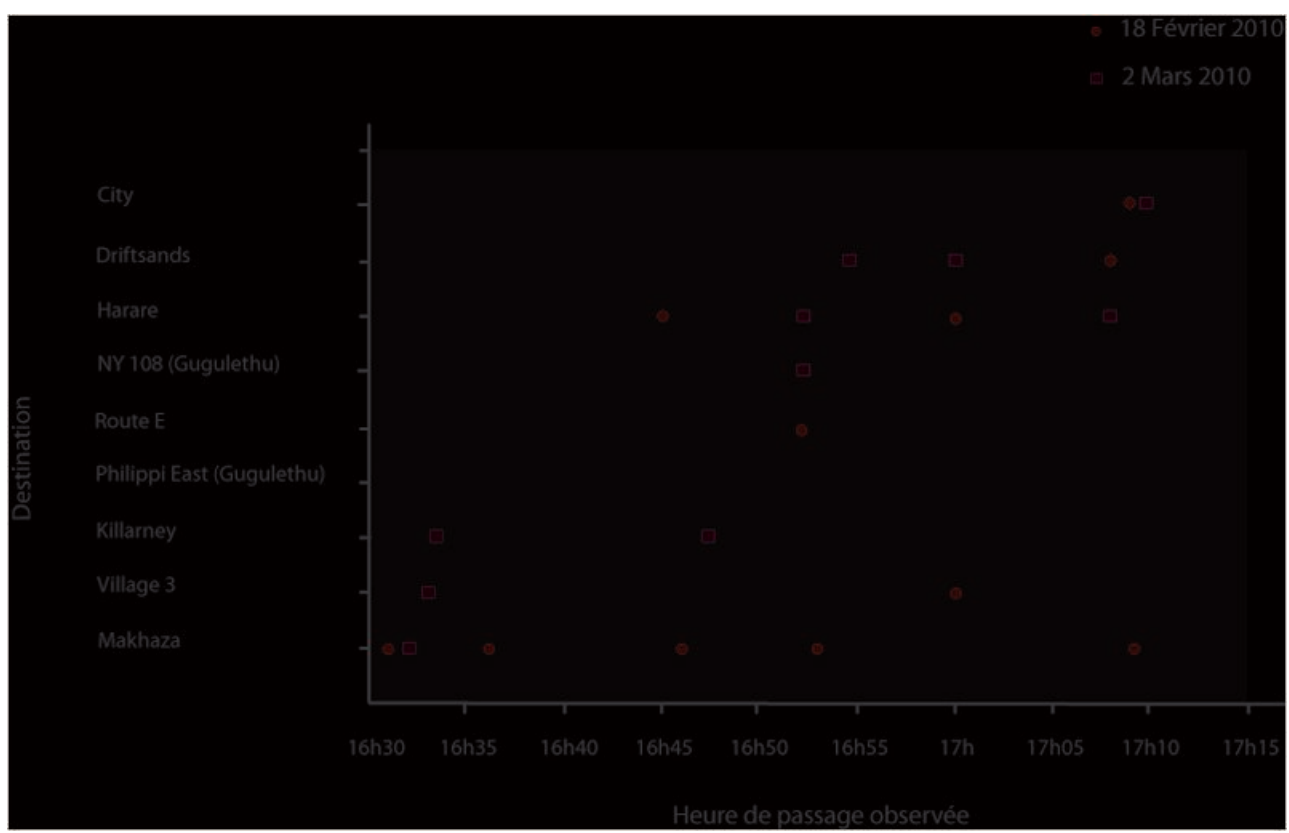

17 Les retards, généralement mal perçus par les employeurs, peuvent être déduits du salairede l'employée. La plupart des maids effectuent ensuite un ou plusieurs changements ; elles prennent soit un autre bus, soit un autre mode de transport. Ces retards, la multimodalité imposée et le peu de bus disponibles contraignent les maids à s'adapter (encadré 1, illustration 4). 


\section{Encadré 1 - Les stratégies mises en place par les maids}

Les entretiens individuels avec les maids ont été l'occasion de comprendre les parcours quotidiens et les stratégies individuelles mises en œuvre pour optimiser le temps de transport. Cette typologie révèle une grande diversité de situations et les stratégies communes à ces femmes.

- Portia travaille à Parklands depuis un an et demi et habite à Site B, à Khayelitsha. Il n'y a pas de bus pour aller de Khayelitsha à Parklands, elle marche donc tous les matins 20 minutes pour être à 5 h30 à la gare de Khayelitsha, où elle prend le bus. Elle arrive à $6 \mathrm{~h} 30$ à Bayside, où elle prend un minibus pour Parklands. Elle en a pour au moins 20 minutes et son travail commence à 7 h30. Portia paie sa clipcard hebdomadaire, mais ses employeurs ont accepté de prendre à leur charge les trajets de minibus.

- Cynthia travaille à Sunningdale depuis 10 ans et habite à Harare, à Khayelitsha. Il lui faut 2 heures pour se rendre sur son lieu de travail. Elle prend un premier bus à $6 \mathrm{~h} 30$ pour Killarney, et de là un autre bus pour Atlantis. Elle arrive au travail pour $9 \mathrm{~h}$. Le soir elle finit son travail à $16 \mathrm{~h}$, prend le bus de $16 \mathrm{~h} 15$ et arrive chez elle généralement vers $19 \mathrm{~h}$.

- Eunice vient de Lower Crossroads, un des townships du Cap, et elle travaille depuis 3 ans chez plusieurs employeurs tous domiciliés à Table View. Elle part le matin à $6 \mathrm{~h}$ de Crossroads, arrive à $8 \mathrm{~h}$ à Killarney puis elle prend un minibus pour arriver à $8 \mathrm{~h} 30$ sur son lieu de travail. Elle quitte son travail à $16 \mathrm{~h}$ et arrive chez elle aux alentours de 18h30. Elle utilise une clipcard hebdomadaire mais souvent elle n'a pas assez d'argent pour prendre le minibus. Il n'y a pas de train pour faire son trajet mais de toute façon le train n'est pas sûr à cause des gangsters, tandis que le bus est sûr.

- Carmen vient de Heideveld et travaille à Sunningdale. Elle part à $7 \mathrm{~h}$ d'Heideveld en bus et prend un autre bus à Killarney qui l'emmène à Sunningdale où elle arrive à $9 \mathrm{~h}$. Sinon elle vient en bus jusqu'à Bayside puis elle prend un minibus. Mais avec la clipcard le bus est moins cher que le minibus. De toute façon Carmen ne prend pas le train, elle déteste le train (elle s'est déjà faite agressée violemment dans le train).

- Malli vit à Khayelitsha, et elle est domestique à Parklands. Elle part à $6 \mathrm{~h}$ de Khayelitsha, prend un bus pour être à $7 \mathrm{~h} 30$ à Bayside puis elle marche jusqu'à Parklands où elle arrive vers $8 \mathrm{~h}$. Elle a une clipcard hebdomadaire mais parfois elle prend le minibus si elle a oublié sa clipcard. Elle a donc le choix entre le bus et le minibus, mais ce n'est pas vraiment un choix puisque le minibus est trop cher. Les trains eux sont bondés, remplis de voleurs. Mais il n'y a pas assez de bus, il en faut plus sur la ligne de Khayelitsha, le matin tous les gens sont debout. 


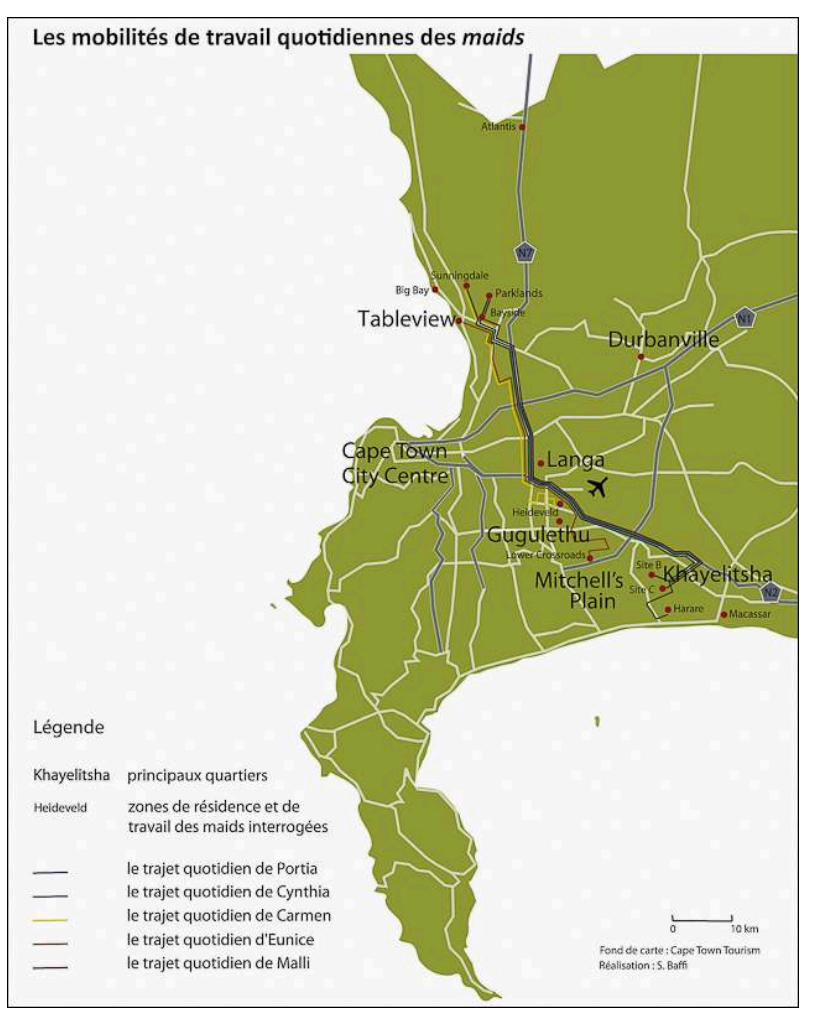

Source : Cape Town Tourism (fond de carte) ; réalisation : S. Baffi.

À ce budget temps s'adjoint un investissement financier. En 2001, Turok estimait que les ménages sud-africains consacraient plus de $10 \%$ de leurs revenus aux transports. Les maids, comme les usagers du train, achètent des clipcards. Ces cartes, valables sur une seule ligne, représentent la seule forme de subvention accordée aux travailleurs par l'État qui verse chaque année des subventions aux compagnies de bus et de trains pour prendre en charge la différence entre le prix des tickets à l'unité et celui des cartes hebdomadaires ou mensuelles. La plupart des maids achètent des cartes hebdomadaires plutôt que mensuelles car elles ne peuvent réunir la somme nécessaire pour payer leur titre de transport pour le mois entier et achètent le plus souvent leur titre de transport le lundi ou le vendredi, c'est-à-dire leur jour de paye. Les clipcards permettent à ces femmes de ne pas transporter d'argent sur elles, ce qui est à leurs yeux un avantage, essentiellement pour des questions de sécurité, par rapport au minibus.

Les conditions de transport sont elles-mêmes relativement difficiles. Le passage aléatoire des bus se traduit par un temps d'attente variable, qui peut parfois être très long. À l'arrêt de bus où ont été réalisés les entretiens, les conditions d'attente posent visiblement problème. Alors que le manque d'aménagements urbains rend les alentours hostiles, l'abribus en tôle qui matérialise l'arrêt du bus n'abrite que très peu de la pluie et du soleil, avec pour toute assise une barre transversale en métal (illustration 5). Cet abri rudimentaire, qui ne peut protéger que trois ou quatre personnes, est bien loin de suffire à la cinquantaine de maids en moyenne qui attendent tous les jours en heure de pointe (illustration 6). Cependant, si les conditions d'attente constituent un motif de plainte récurrent chez les maids, elles jugent les conditions de voyage à l'intérieur du bus confortables, pratiques et sûres, malgré le manque de sièges, qui amène souvent 
une partie d'entre elles à rester debout pendant la durée du voyage. Pour ces femmes, il semble donc que le transport lui-même ne soit pas un problème mais que ce sont les conditions extérieures non-maitrisées telles que le temps d'attente qui sont de véritables obstacles (encadré 2).

Illustration 5 - Les conditions d'attente des maids à l'arrêt de bus de Bayside

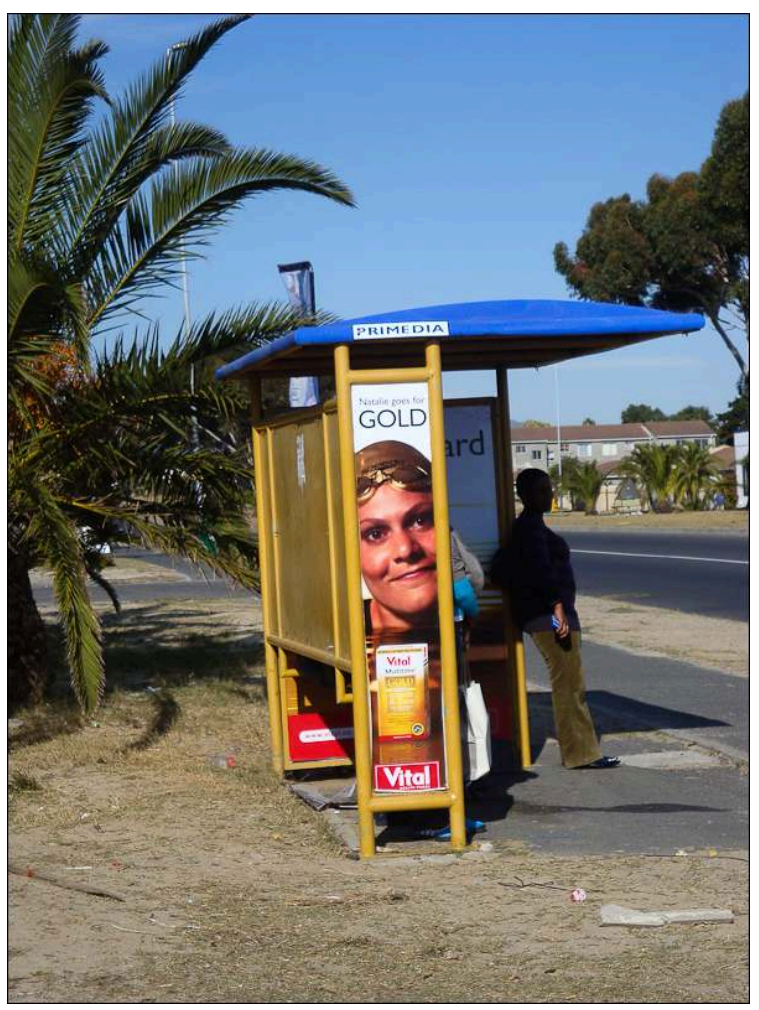

Illustration 6 - Les bus, pris d'assaut par les usagères en heure de pointe

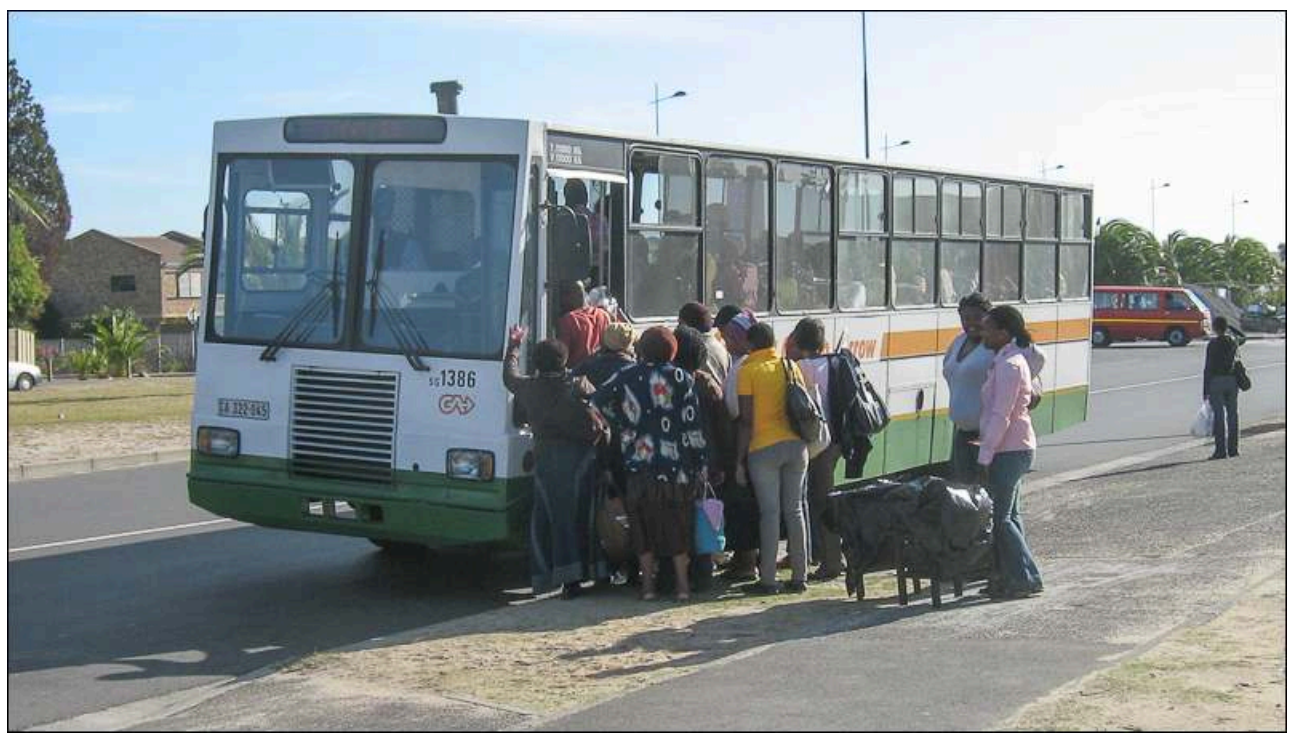

Ici à l'arrêt de Bayside en direction de Macassar. 
Patricia est domestique depuis 6 ans, elle travaille à Parklands et habite à Khayelitsha Down. Tous les jours, elle prend le bus à $5 \mathrm{~h} 30$, car ensuite il est trop plein. Elle effectue un changement à Killarney; à $7 \mathrm{~h} 30$ elle arrive sur son lieu de travail, celui-ci commençant à $8 \mathrm{~h}$. Patricia finit son travail à 15h30, mais comme il n'y a pas de bus entre Parklands et Killarney à cette heure-ci, elle est obligée de payer un minibus avant de prendre le bus de $16 \mathrm{~h}$ à Killarney. Elle arrive à Khayelitsha à 17h. Patricia estime qu'elle n'a pas le choix, ni dans le mode de transport, ni dans le prix. Le bus est cher, mais tant qu'elle peut payer une clipcard, elle est obligée de le prendre. Elle pense néanmoins que la politique de Golden Arrow n'est pas correcte : la plupart des habitants de Khayelitsha utilisent des clipcards, et pourtant les prix augmentent deux fois par an.

Le bus est «safe ", c'est un mode de transport « okay» quant à ses conditions de voyage. Toutefois les conditions d'attente sont très mauvaises :l'abribus ne sert à rien, surtout quand il se met à pleuvoir, et il faut attendre debout. Lorsqu'ils arrivent, les bus sont souvent pleins car le service n'offre pas une fréquence suffisante pour le nombre d'habitants de Khayelitsha qui travaillent dans cette zone. De plus, les bus sont souvent en retard, alors que Patricia «just want to go home ». Et lorsqu'ils sont en retard, les chauffeurs se précipitent. Le problème ce ne sont pas les chauffeurs, qui souvent conduisent bien, mais leurs supérieurs qui exercent une pression trop forte, ce qui les pousse à démissionner.

Malgré ces doléances, les maids ne se positionnent pas comme des « victimes » de leur mode de transport, au contraire. Ce sont bien elles qui choisissent le transport le plus adapté à leurs exigences, que ce soit en terme financier, de budget temps, de confort ou de praticité. Ce n'est pas tant le déplacement en soi qui est contraignant pour ces femmes que les aspects qu'elles ne maîtrisent pas. Le trajet est admis comme étant incompressible, nécessaire, tandis que le retard des chauffeurs et l'irrégularité constituent les véritables motifs de plainte. Il y a en effet mise en place de stratégies de la part de ces utilisatrices qui cherchent à optimiser leurs mobilités à travers un certain nombre de choix. Parmi ces stratégies, on peut noter l'aptitude à grouper les déplacements. Si de nouvelles mobilités émergent en lien avec les évolutions sociales, il n'est pas pour autant question de leur consacrer une part plus importante du budget temps et financier des ménages. Dans le cas des maids, les mobilités de consommation se font presque systématiquement sur le chemin entre lieu de travail et lieu de résidence afin d'utiliser leur clipcard sans avoir à payer de nouveau un titre de transport. Néanmoins, ces femmes sont alors contraintes en ce qui concerne la localisation du mall dans lequel elles se rendent.

Cette mise en place de stratégies dans le but de minimiser l'aspect éprouvant du déplacement est particulièrement révélatrice de la capacité de ce groupe social à organiser ses mobilités en fonction des contraintes qui pèsent sur lui. Les stratégies des maids manifestent effectivement la mobilisation d'un capital de mobilité, qui différencie les déplacements effectués de la capacité des individus à se déplacer, ce que Kaufmann (2007) nomme motilité (in Oppenchaim, 2011). Or, dans la mesure où la mobilité peut être considérée comme une action en vue d'accéder à une ressource, le capital de mobilité varie également différemment en fonction des groupes sociaux (Enaux, Lannoy, Lord, 2011). Par ailleurs, les habitudes, normes et valeurs intériorisées par les individus influencent aussi le potentiel de déplacement. En d'autres termes, l'appropriation est l'une des facettes de la motilité, qui permet de considérer la mobilité comme une pratique (Oppenchaim, 2011). Dans le cas des maids, on est en effet 
en présence d'une forte capacité à se déplacer, alliée à une appropriation de l'espace urbain qui font des déplacements de ces femmes une pratique quotidienne de la métropole. On observe donc une production sociale et une pratique de l'espace urbain par «le bas», par un groupe d'individus capables de naviguer entre les contraintes économiques de la ville émergente et les déterminations raciales de la ville postapartheid.

\section{L'espace du transport, un sas social}

L'observation des pratiques des usagers au sein même des transports collectifs permet d'analyser à une échelle plus fine les dynamiques mises en évidence précédemment, afin de les préciser et d'en révéler d'autres. Les conditions à bord des véhicules sont à mêmede nous informer sur le fonctionnement de la société sud-africaine en général. Les pratiques des trois modes de transport sont tout autant exclusives qu'excluantes, stigmatisant aussi bien les usagers des transports que ceux qui n'en sont pas familiers. À cet égard, on peut parler de l'espace du transport comme d'un microcosme, reflet des dynamiques qui façonnent la société en général, "le territoire qui manifeste l'interaction généralisée qui anime le monde ", (Lombard, Steck, 2004). L'espace du transport devient un lieu, une "cellule spatiale", ce qui est d'autant plus vrai en Afrique du Sud où les transports ont été l'un des instruments de la fabrique de la ville d'apartheid, comme l'explique Voges (1984): "The location of residential areas has a marked influence, therefore, on the accessibility of different groups of people».

Plus encore, en tant qu'élément urbain, le transport contribue à construire le rôle de l'usager au sein de la société (Yago, 1983). Dans cette mesure, l'étude de la pratique des transports publics par ses usagers éclaire dans le même temps la position de ces individus dans la ville. Or, dans le cas des maids, l'espace du transport est associé à des connotations positives. Si elles utilisent fréquemment des adjectifs négatifs pour parler des éléments extérieurs (condition d'attente, irrégularité des horaires, coûts), le trajet lui-même est considéré comme agréable, confortable et chaleureux. Le moment du transport constitue en effet pour elles une pause dans la journée, souvent la seule, entre leur travail et leur vie de famille. Lorsqu'elles rentrent ces femmes ont souvent une famille à charge composée des enfants mais également des petits enfants, et c'est à elles qu'incombent l'organisation de la vie de famille et les tâches ménagères. L'espace du bus devient donc un sas entre deux structures sociales où la place de ces femmes n'est pas évidente, un espace de sécurité où elles se retrouvent entre amies. L'espace du transport devient alors un espace de transition.

Plus généralement, les transports publics correspondant à des catégories d'usagers spécifiques, le plus souvent appartenant aux lower income ou lower middle class et résidant dans les townships, l'espace transitoire du transport est, pour ces usagers, un lieu d'identification sociale et culturelle qui n'existe pas forcément en dehors du lieu de résidence. Ainsi, un certain nombre de codes tacites régulent le fonctionnement à bord des transports, en particulier des minibus. Un certain nombre de pratiques rituelles, telles que le passage de la monnaie, la relation au chauffeur et aux autres passagers ou la demande d'un arrêt, ponctuent le trajet et nécessitent la participation de chaque passager, qui devient ainsi garant du bon fonctionnement au sein du minibus, microcosme de la communauté à laquelle il appartient. Le trajet est ponctué par les différents arrêts demandés par les passagers qui préviennent toujours quelques 
centaines de mètres avant l'endroit où ils désirent s'arrêter. Les stops correspondent le plus souvent à des points de repères connus de tous: enseignes commerciales, bâtiments publics, points de correspondance vers d'autres transports. Dans le cas du bus, le glissement d'usage d'un transport public à un transport de travailleurs aboutit à une utilisation quasi privée de ce service. Ceci tient en partie au fait que ce mode de transport a été largement approprié par une catégorie d'usagers, dont les maids sont un bon exemple, faisant de ce service un mode de transport communautaire.

Plus précisément encore dans le cas de l'utilisation du bus par les maids, on peut parler d'une appropriation genrée. L'une des préoccupations récurrentes évoquées par les maids est celle de la sécurité, manifeste lors des entretiens par l'occurrence du mot «safe » en parlant des bus, à l'inverse des trains et des minibus. Il est en effet frappant de remarquer que le bus se distingue comme un mode de transport essentiellement féminin. La sécurité est assurée d'une part grâce aux clipcards, qui autorisent les femmes à se déplacer sans argent sur elles et limite le risque d'agression des chauffeurs. Par ailleurs, une fois à bord, il n'y a pas de risque de violence physique ou verbale, choses fréquentes dans le minibus et le train. Hormis le chauffeur, les usagers sont essentiellement des femmes et l'accès à l'intérieur du bus se restreint à une unique entrée relativement étroite, face à la cellule grillagée du chauffeur. Il faut en effet rappeler que les lieux de transports sont généralement associés à la criminalité (Lombard, Steck, 2004) et plus particulièrement en Afrique du Sud à la violence, à laquelle les femmes sont particulièrement exposées. Parmi la vingtaine de maids interrogées, une d'entre elle a expliqué ne plus prendre le train depuis qu'elle y avait été violée. Les stratégies mises en place par les maids intègrent donc également des stratégies de survie, alors que le voyage quotidien représente souvent la seule apparition de ces femmes dans la sphère publique, recluses dans la sphère privée au travail comme dans leur vie de famille (Destremeau, Lautier, 2002).

L'exemple de l'appropriation du bus par les maids démontre certes leurs stratégies face au transport, mais il est également manifeste de la position de ces femmes au sein de la société sud-africaine. En plus de leur travail, les femmes continuent d'assurer les tâches ménagères et familiales et vont être plus susceptibles d'effectuer des trajets à buts multiples (Potgieter, Pillay, Rama, 2006). Plus généralement, les femmes utilisent plus fréquemment les transports publics, tandis que les hommes préfèrent l'utilisation d'un véhicule privé. Les femmes sont donc plus souvent et plus longtemps dans les transports publics, alors que cet espace est pour elles souvent chargé de craintes (Cresswell, Priya Uteng, 2008).

27 Toutefois, R. Pain (2001) précise dans ses travaux que loin de vivre l'espace de façon passive, les femmes le produisent, le définissent et le revendiquent de façon active. L'auteure parle des pratiques spatiales quotidiennes des femmes comme de pratiques de résistance qui contribuent à l'évolution de l'espace public à l'échelle de la société. Ainsi, on retrouve ici encore ce paradoxe de femmes obligées de déployer des stratégies - notamment à travers leurs mobilités - pour exister socialement, faisant d'une contrainte un choix volontariste, une production spatiale. Finalement, si l'on peut parler du transport comme d'un lieu (Lombard, Steck, 2004), il semble que l'on soit plutôt en présence d'un entre-lieux pour ces usagères qui naviguent entre des espaces sociaux où leur position est constamment négociée. Cet entre-lieux, ce sas social, apparaît alors comme un espace d'expression, de sociabilité et de pratiques créatives. De ce fait, la position de ces usagères au sein des transports reflète l'ambigüité de leur 
position au sein de la société, non seulement en fonction de déterminations sociales ou raciales, mais aussi en fonction de la détermination du genre.

\section{Conclusion}

Le système de transport public actuel de la métropole du Cap contribue tout autant qu'il palliele dysfonctionnement hérité de l'apartheid et renforcé par le processus d'émergence. La qualité du service ne permet pas pour l'instant de diversifier la clientèle des transports publics, qui reste associée aux catégories des lower income et lower middle class résidant encore le plus souvent dans les townships, tandis que les populations plus aisées peuvent faire le choix du transport privé. Les mobilités de ces usagers demeurent largement contraintes par la rigidité du réseau et le service aléatoire. Toutefois ces usagers, dont les maids sont un bon exemple, sont souvent en mesure de faire un certain nombre de choix qui leur permettent de développer des stratégies de déplacement en vue de rentabiliser leur capital de mobilité. De cette façon, ils produisent une pratique de la ville qui leur est propre et négocient leurs mobilités au sein d'un espace urbain complexe, marqué par les frontières sociales et raciales. Enfin, l'espace du transport lui-même apparaît comme un microcosme de la société. S'il stigmatise une catégorie d'usagers, il est aussi le lieu d'échanges sociaux riches et variés, lieu d'appropriation de la ville pour une population longtemps exclue de la ville d'apartheid (Davies, 1981) et qui cherche à s'inscrire dans le modèle de la ville émergente. Ainsi, la réalisation du BRT dans cette métropole émergente présente un enjeu majeur si elle permet la reconquête d'une urbanité confisquée tout en promouvant l'idéal démocratique d'une intégration métropolitaine.

29 Yago G., 1983. The sociology of transportation. Annual Revue of Sociology, vol. 9, p. 171-190.

\section{BIBLIOGRAPHIE}

Anderfuhren M., 2002. Mobilité professionnelle des domestiques au Brésil (Nordeste) : une logique complexe. Tiers-Monde, vol. 43, n 170, p. 265-285.

Baffi S., 2010. Les transports urbains au Cap, entre fragmentation et structuration, le cas du Cap, Afrique du Sud. Mémoire de recherche de Master 1, Université Paris 1 Panthéon-Sorbonne.

Behrens R., Wilkinson P., 2003. Housing and urban passenger transport policy and planning in South African cities: a problematic relationship ?, in Harrison, P., Huchzermeyer, M., Mayekiso, M., Confronting fragmentation : housing and urban development in a democratising society. Cape Town, University of Cape Town Press, p. 154-174.

Bénit C., 1998. Gouvernement urbain et production de la ségrégation : quelles leçons de la « ville d'apartheid ». Une comparaison Johannesbourg-Los Angeles. Revue européenne de migrations internationales, vol. 14, $\mathrm{n}^{\circ} 1$, p. 159-192. 
Bénit C., Morange M., 2004. Les domestiques, la ville et l'accès à l'emploi au Cap et à Johannesbourg : logiques de proximité et logiques de réseau. Tiers-Monde, vol. 179, $\mathrm{n}^{\circ} 45, \mathrm{p}$. 539-565.

Bret B., 2011. Un questionnement géographique sur les pays émergents. Bulletin de l'Association de Géographes Français, vol. 88, n³ 3, p. 227-240.

Cresswell T., Priya Uteng T., 2008. Gendered mobilities. Aldershot, England, 270 p.

Davies R. J., 1981. The spatial formation of South African City. Geojournal, vol. 2, n² 2, p. 59-72.

Destremeau B., 2002. L'émergence d'un marché du travail domestique au Yémen : une étude sur Sana'a. Tiers-Monde, vol. 43, n²170, p. 327-351.

Destremeau B., Lautier B., 2002. Femmes en domesticité. Les domestiques du Sud, au Nord et au Sud. Tiers-Monde, vol. 43, n 170, p. 249-264.

Enaux C., Lannoy P., Lord S., 2011. Les mobilités éprouvantes. Regards sur les pénibilités de déplacements ordinaires. Articulo-Journal of urban research, $\mathrm{n}^{\circ} 7$.

Houssay-Holzschuch M., 1996. Mythologies territoriales en Afrique du Sud. Paris, CNRS, 104 p. Integrated Transport Plan, 2006. Cape Town municipality.

Lombard, J., Steck B., 2004. Quand le transport est d'abord un lieu! Autrepart, n 32, vol. 4, p. 3-19.

Musil C., Ninot O., Baffi S., Drevelle M., 2012. Évolutions des systèmes de transport urbain en périphérie du Cap et de Hanoi : entre pragmatisme et ambitions métropolitaines, à paraître

Nitrass N., 2002. Unemployment, employment and labour force participation in Khayelitsha and Mitchell's Plain. CSSR, working paper $\mathrm{n}^{\circ} 12,31 \mathrm{p}$.

Naudé W., 2008. Is there a spatial mismatch in South Africa's labour market ? Cities, vol. 5, p. 268-276.

Oppenchaim N., 2011, La mobilité comme accessibilité, disposition et épreuve : trois paradigmes expliquant le caractère éprouvant des déplacements à Paris. Articulo-Journal of urban research, $\mathrm{n}^{\circ} 7$.

Pain, R., 2001. Gender, Race, Age and fear in the city. Urban studies, vol. 38 n 5-6, p. 899-913.

Potgieter C-A., Pillay R., Rama S., 2006. Women, development and transport in rural Eastern Cape. South Africa, Human Science Research Council Press, Cape Town.

Statssa, 2010. Monthly earnings of South Africans.

Statssa, 2012. Quarterly Labour Force Survey.

Turok I., Watson V., 2001. Divergent development in South African cities: strategic challenges facing Cape Town. Urban Forum, vol. 12 n² 2, p. 119-138.

Urry J., 1999. Automobility, car culture and weightless travel : a discussion paper. Department of Sociology, Lancaster University, 16 p.

Varel A., 2002. Itinéraires du travail domestique en Inde : les filles d'Erayiur. Tiers-Monde, vol. 43, $\mathrm{n}^{\circ} 170$, p. $353-371$

Voges E. M., 1984. Accessibility, transport and the spatial structure of South African cities : an historic perspective. National Institute for Transport and Road research, Pretoria, $47 \mathrm{p}$. 


\section{NOTES}

1. Les maids appartiennent à la catégorie socio-professionnelle des domestic workers, les domestiques. Elles se distinguent des autres professions qui appartiennent à cette catégorie (les cuisinières, femmes de chambres, nourrices) en assurant les tâches ménagères au domicile des particuliers ou entreprises.

2. L'étude de cas concernant les maids a été réalisée au printemps 2010 dans le cadre du mémoire de recherche de Master 1 . Une vingtaine d'entretiens qualitatifs semi-dirigés ont été réalisés auprès des maids à l'arrêt de bus Golden Arrow de Bayside.

3. Le programme lancé par le gouvernement en 1999, baptisé Taxi Recapitalisation Programme, visait à formaliser le secteur des minibus qui assurait de façon informelle près de $50 \%$ du transport urbain pendant l'apartheid. Ce programme, qui incluait l'enregistrement des chauffeurs et le renouvellement des véhicules n'a jamais été réellement achevé dans la mesure où un certain nombre des acteurs du secteur des minibus n'a pas souhaité y souscrire, ou a détourné l'argent alloué pour acheter de nouveaux véhicules (Baffi,2010).

4. Les bas revenus, selon la nomenclature socio-économique sud-africaine.

5. Les grants sont des subventions versées mensuellement aux personnes âgées, handicapées, aux familles d'accueil, aux personnes médicalement dépendantes, comme allocation familiale et aide sociale. Cette subvention concerne majoritairement les habitants des townships.

6. Les classes moyennes inférieures, selon la nomenclature socio-économique sud-africaine.

\section{RÉSUMÉS}

La ville du Cap, à l'image des autres villes sud-africaines, se développe selon le double modèle de la ville post-apartheid et de la ville émergente. Cette évolution implique des changements démographiques, sociaux et politiques importants, particulièrement visibles dans l'espace urbain en raison du spatial mismatch qui continue de caractériser la ville et d'isoler les populations des townships des opportunités de la ville, en particulier des bassins d'emplois. Cela se traduit par des mobilités longues et fastidieuses pour les employés des catégories sociales inférieures, alors que le système de transport en commun demeure encore largement hérité de la période d'apartheid. L'étude des mobilités des maids permet d'éclairer ces mutations socio-spatiales, notamment par l'observation de leur pratique du transport, des stratégies qu'elles déploient pour se déplacer et de leur expérience de l'espace du transport.

The city of Cape Town, like other cities in South Africa, is developing towards the dual model of the post-apartheid city and the emerging city. This evolution involves important demographic changes, as well as social and political. Those changes are especially visible in the urban space due to the spatial mismatch which still characterizes the city and keeps on isolating township dwellers from benefiting the city's opportunities, especially in terms of labour. It results in long and fastidious mobilities for low-income populations, whereas the transport system is still largely inherited from the apartheid era. The study of the maids' mobilities enables to enlighten these socio-spatial changes, especially through the observation of their practice of the transport network, the strategies they adopt to move and through their experience of the transport space. 
INDEX

Mots-clés : Le Cap, maids, transport en commun, ville émergente, ville post-apartheid

Keywords : Cape Town, emerging city, maids, post-apartheid city, Public transport

Thèmes : Sur le Champ - Sur le Terrain

\section{AUTEUR}

\section{SOLÈNE BAFFI}

Solene Baffi, solene.baffi@gmail.com, est doctorante en géographie à l'Université Paris 1 Panthéon Sorbonne et au laboratoire Géographie-cités. Son travail de thèse porte sur les interactions ville-transport en Afrique du Sud. 